\title{
Proposta de Mapa Interativo e Colaborativo para Ecossistemas de Inovação: Um Estudo de Caso Baseado no Estado do Ceará
}

\section{Rommel Castro ${ }^{1}$, Allysson Allex Araújo ${ }^{2}$ Pamella Soares $^{1}$, Matheus Paixão ${ }^{1}$, Paulo Henrique M. Maia ${ }^{1}$ e Jerffeson Souza ${ }^{1}$}

${ }^{1}$ Programa de Pós-Graduação em Ciência da Computação (PPGCC) Universidade Estadual do Ceará (UECE) - Fortaleza, Ceará - Brasil

${ }^{2}$ Grupo de Estudos em Sistemas de Informação e Inovação Digital (GESID) Universidade Federal do Ceará (UFC) - Crateús, Ceará - Brasil

\begin{abstract}
The collaboration between agents within an innovation ecosystem proves to be an important socioeconomic agenda to be explored. Among the initiatives that contribute towards this issue, the adoption of interactive maps stands out. However, regardless of their relevance, these projects still face a lack of empirical Human-Computer Interaction evaluation. Hence, we aim to develop and evaluate an interactive and collaborative map exploring the state of Ceará as a case study that, despite its recognized prominence, does not have an open source initiative at a state scale for mapping its agents. Under a qualiquantitative methodological scope, we justify this work by framing it within the dynamics of innovation diffusion. In addition to providing an open source solution in line with the $4 C$ Collaboration Model, this work pioneers in conducting an empirical evaluation of User Experience, Usability, and Perception of Value in the context of a Brazilian innovation ecosystem.
\end{abstract}

Resumo. A colaboração entre os agentes que entrelaçam um ecossistema de inovação demonstra-se uma importante pauta socioeconômica a ser explorada. Dentre as iniciativas que contribuem para tal consolidação, destaca-se a adoção de mapas interativos que, apesar de relevantes, enfrentam uma lacuna empírica quanto à avaliação de elementos associados à Interação HumanoComputador. Diante dessa oportunidade, esta pesquisa objetiva desenvolver e avaliar um mapa interativo e colaborativo explorando o estado do Ceará como estudo de caso que, mesmo reconhecidamente proeminente, ainda não dispõe de iniciativas intrinsecamente open source, de expressão estadual e colaborativa para mapeamento dos seus agentes. Sob um escopo metodológico qualiquantitativo, este trabalho se justifica devido ao seu enquadramento dentro da dinâmica de difusão da inovação. Portanto, além da disponibilização de uma solução open source aderente ao Modelo $4 C$ de Colaboração, este trabalho contribui, de forma pioneira, na condução de um experimento para avaliação empírica de Experiência do Usuário, Usabilidade e Percepção de Valor no contexto de um ecossistema de inovação brasileiro.

\section{Introdução}

Compreende-se o processo de inovação como um ativo importante para o crescimento socioeconômico [Tidd and Bessant 2015]. Sabe-se que parcela do conhecimento oriundo 
deste processo é transferido por meios de comunicação, porém, outra parte dele, que permanece tácito, somente é transferido por meio das relações sociais. Assim, torna-se preponderante o relacionamento entre os diferentes agentes econômicos como incentivo para o crescimento da capacidade inovativa [Spinosa and Krama 2014]. A conscientização oriunda dos relacionamentos entre os agentes econômicos potencializa as interações e, consequentemente, favorece o surgimento de redes de cooperação [Lemos 2009]. Nesse sentido, tem-se o advento dos ecossistemas de inovação, os quais emergem como ambientes de intensa cooperação entre diferentes entidades de diversos setores com o intuito de geração de conhecimento [Jin-fu 2010].

O fortalecimento dos ecossistemas de inovação se revela de grande importância para o crescimento do país, pois, no Brasil, se presencia, por parte das empresas, um movimento de ampliação do percentual dedicado à aquisição de $\mathrm{P} \& \mathrm{D}$ de institutos de pesquisa ou outras empresas em contraste à redução do investimento interno [IPEA 2016]. Por isso, se torna relevante a difusão dos integrantes e das propostas de valor presentes nesses ecossistemas, tendo em vista que, a partir desta divulgação, pode-se engajar novos participantes, potencializar iniciativas, atrair novos investidores, dentre outros benefícios.

Dentre as iniciativas que podem contribuir para a consolidação de ecossistemas de inovação, pode-se destacar a oportunidade de geovisualização através de mapas interativos [MacEachren and Kraak 2001]. A adoção de mapeamentos em ecossistemas de inovação tem se demonstrado amplamente adotado em outros estados do Brasil, como, por exemplo, Minas Gerais $\varsigma^{1}$, Alagoas ${ }^{2}$ e Santa Catarina ${ }^{3}$, Todavia, verifica-se que, sob um ponto de vista científico, há uma lacuna concernente à avaliação sistemática e empírica de elementos relacionados à Interação Humano-Computador (IHC) em propostas com essa finalidade, incluindo questões relacionadas a aspectos colaborativos na realização de tarefas, Experiência de Usuário (em inglês User eXperience ou UX) e Usabilidade.

A partir da oportunidade previamente contextualizada, este trabalho objetiva desenvolver e avaliar uma solução baseada em geovisualização para ecossistemas de inovação alicerçada em duas dimensões. Primeiro, sob a dimensão interativa, opta-se pelo desenvolvimento do mapa geográfico enquanto solução web, tendo em vista a facilidade de extensão dos componentes e produtividade para desenvolvimento de protótipos para validação. Nesse sentido, tem-se, ainda, este projeto como open source, de tal modo que se possa incentivar a manutenção e desenvolvimento de novas funcionalidades a partir da própria comunidade que compõe o ecossistema, tendo, inclusive, a possibilidade do projeto ser estendido e adotado por outras regiões. Segundo, quanto à dimensão colaborativa, tem-se uma concepção da solução aderente ao Modelo 4C de Colaboração, o qual fundamenta-se nos pilares: comunicação, coordenação, colaboração e cooperação [Costa et al. 2014]. Adicionalmente, este projeto agrega funcionalidades de teor colaborativo não contempladas nas iniciativas de mapeamento mencionadas anteriormente, como, por exemplo, integração de eventos ao mapa e demarcação de comunidades.

Portanto, este trabalho se justifica devido ao seu escopo pautado na dinâmica de difusão da inovação o qual fundamenta a inovação por meio de canais de comunicação e pelos membros deste sistema social [Schumpeter et al. 1939]. Sob o ponto de vista meto-

\footnotetext{
${ }^{1}$ http://www.simi.org.br/mapa

${ }^{2} \mathrm{http} / / /$ mapainovacao.secti.al.gov.br/

${ }^{3}$ https://via.ufsc.br/mapas-da-via/
} 
dológico, esta pesquisa se enquadra a partir de um estudo de caso baseado no ecossistema de inovação do estado do Ceará, o qual vem se destacando no cenário brasileiro e, atualmente, não dispõe de nenhuma solução com o escopo aqui delineado. Assim, como principais contribuições, tem-se a disponibilização de uma solução funcional e open source que permite o mapeamento colaborativo de um ecossistema de inovação. Adicionalmente, relata-se, de forma pioneira, a realização de um experimento quali-quantitativo com 20 potenciais usuários para avaliação de UX, Usabilidade e Percepção de Valor no contexto de um sistema colaborativo baseado em geovisualização de ecossistemas de inovação.

\section{Procedimentos Metodológicos}

\subsection{Caracterização da Pesquisa}

O presente trabalho é pautado pelo tipo de pesquisa exploratória, sob a forma de um estudo de caso [Yin 2017]. Nesse sentido, o estudo de caso explorado neste trabalho consiste no ecossistema de inovação do estado do Ceará o qual vem avançando numa posição de destaque em termos regionais e nacionais. Conforme levantamento realizado em 2020 pela Federação das Indústrias do Estado do Ceará, o Ceará ficou na $13^{a}$ posição no ranking nacional de inovação e, no Nordeste, o Estado ficou na $3^{\text {a }}$ colocação, atrás de Pernambuco $\left(11^{\circ}\right)$ e da Paraíba $\left(12^{\circ}\right)$ [do Nordeste 2020]. Tal enfoque converge, portanto, com o objetivo deste trabalho, que é a formulação e avaliação de um produto [Wazlawick 2009]. Projetada como uma aplicação web, a solução proposta almeja facilitar o mapeamento de agentes e eventos relevantes para o cenário de inovação do estado e, consequentemente, aproximar e fortalecer as comunidades. Assim, além de apresentar a solução e as potencialidades identificadas, faz-se necessário um diagnóstico quanto i) aos aspectos colaborativos, ii) à UX, iii) à Usabilidade e, finalmente, iv) à Percepção de Valor do sistema. Como ilustrado na Figura 1, optou-se por um plano metodológico quali-quantitativo dividido em quatro etapas, conforme sintetizado a seguir.

\begin{tabular}{|c|c|c|c|}
\hline ETAPA 1 & ETAPA 2 & ETAPA 3 & ETAPA 4 \\
\hline $\begin{array}{l}\text { Análise de dados } \\
\text { secundários }\end{array}$ & $\begin{array}{c}\text { Desenvolvimento } \\
\text { da solução }\end{array}$ & $\begin{array}{c}\text { Avaliação } \\
\text { comparativa }\end{array}$ & $\begin{array}{l}\text { Experimentos } \\
\text { com usuários }\end{array}$ \\
\hline $\begin{array}{l}\text { - Compreensão do domínio } \\
\text { - Mapeamento dos requisitos } \\
\text { - Identificaçãa de premissas do } \\
\text { Modelo 4C de Colaboração }\end{array}$ & $\begin{array}{l}\text { - } 4 \text { meses (março/2020 - julho/2020) } \\
\text { - Aplicação web } \\
\text { - HTML, CSS, JavaScript, } \\
\text { Bootstrap, jQuery }\end{array}$ & $\begin{array}{l}\text { - Comparação baseada no } \\
\text { Modelo 4C com outras } \\
\text { iniciativas }\end{array}$ & $\begin{array}{l}\cdot \text { UX } \\
\text { - Usabilidade } \\
\text { - Percepção de Valor }\end{array}$ \\
\hline
\end{tabular}

Figura 1. Procedimentos metodológicos.

Na primeira etapa, realizou-se uma análise de dados secundários com o objetivo de obter uma compreensão ampla sobre elementos teóricos e práticos pertinentes à proposta. Para tal processo, houve inicialmente uma análise bibliográfica específica sobre a Teoria da Inovação [Schumpeter et al. 1939], incluindo a formação de ecossistemas e os agentes envolvidos [Spinosa and Krama 2014]. Visando a definição do escopo do projeto e a adoção de referências de mapas interativos, realizou-se uma busca manual no Google e Google Scholar através de palavras-chave objetivando identificar iniciativas brasileiras que estejam em operação. Como resultado, identificou-se três projetos (Minas Gerais, Alagoas e Santa Catarina) os quais foram submetidos à uma análise de benchmarking via observação não participante tanto em relação aos aspectos positivos e possíveis funcionalidades, quanto limitações a serem contornadas. Em especial, dentre as oportunidades 
observadas, identificou-se as premissas do Modelo 4C de Colaboração [Costa et al. 2014] como diretrizes valiosas a serem incorporadas na concepção do projeto aqui proposto.

Após uma compreensão do domínio e dos requisitos fundamentais mapeados (incluindo os diferenciais), deu-se início a segunda etapa, na qual consistiu no desenvolvimento da solução sob a forma de uma aplicação web. Tal etapa de desenvolvimento teve como enfoque contemplar a dimensão interativa da solução e durou cerca de quatro meses, tendo início no mês de março e sendo finalizado no mês de julho de 2020.

Na terceira etapa conduziu-se, baseando-se em [Melo et al. 2017], uma avaliação comparativa à luz do Modelo 4C [Costa et al. 2014]. Dessa forma, investigou-se a aderência da solução proposta, frente aos pilares de comunicação, coordenação, colaboração e cooperação quando comparada à outras iniciativas de mapeamento, que serviram de benchmarking para este trabalho, neste caso: Minas Gerais, Santa Catarina e Alagoas. Tal avaliação, de cunho observacional, foi pautada pela verificação da quantidade de componentes que dão suporte a cada dimensão do Modelo 4C.

A quarta etapa refere-se a realização de um experimento baseado em teste com usuários [Rogers et al. 2013] para avaliação de UX, Usabilidade e Percepção de Valor. Inspirado em [Valentim et al. 2015], tal experimento foi baseado na realização de um conjunto de tarefas a fim de se obter uma consciência do padrão de usabilidade. As técnicas adotadas para coleta de dados foram observação participante e questionários semi-estruturados. Para a Avaliação da $U X$, adotou-se o questionário AttrakDiff [Hassenzahl 2004] devido à sua capacidade de avaliar a atratividade através de aspectos pragmáticos, hedônicos, de estímulo e atratividade de uma aplicação. O Teste de Usabilidade, por sua vez, baseou-se na contabilização dos erros cometidos das tarefas sugeridas [Winckler and Pimenta 2002]. A Avaliação da Percepção de Valor se deu através das respostas de um questionário composto por cinco perguntas (as três primeiras em uma escala Likert de cinco itens e as duas últimas abertas). Detalhes sobre os questionários encontram-se disponíveis na página de suporte deste artigo [Castro et al. 2020]. Após a conclusão da coleta de dados, tem-se, finalmente, a interpretação quali-quantitativa dos resultados, incluindo as respectivas análises experimentais de elementos relacionados à UX, Usabilidade e Percepção de Valor. Em específico, as questões abertas foram interpretadas com o apoio da técnica Análise de Conteúdo em busca de significados inferenciais e interpretativos [Bardin 2010].

\subsection{Desenho do Experimento}

A seleção dos participantes se deu através de dois critérios específicos: (i) demonstra algum envolvimento profissional no ecossistema de inovação do estado (por exemplo, trabalha em startups, articulador, pesquisador, etc) e (ii) maior de 18 anos. O recrutamento dos convidados se deu em duas fases. Inicialmente, identificou-se os possíveis participantes via snowball sampling através do LinkedIn e rede de contato dos autores, e, em seguida, enviaram-se os convites individualmente via e-mail. Neste convite, existia uma breve descrição do projeto, de seus riscos e benefícios, das etapas da coleta de dados e do tempo de duração da coleta. O experimento ocorreu entre os dias 24/07/2020 a 31/08/2020. Ao total, dos 41 convites enviados, 20 aceitaram participar do estudo. A Figura 2 apresenta uma síntese caracterizando todos os participantes, o que evidencia uma variedade de perfis profissionais e formações acadêmicas, contribuindo, assim, para uma maior representatividade de perspectivas. 
(a) Formação/Atuação Profissional

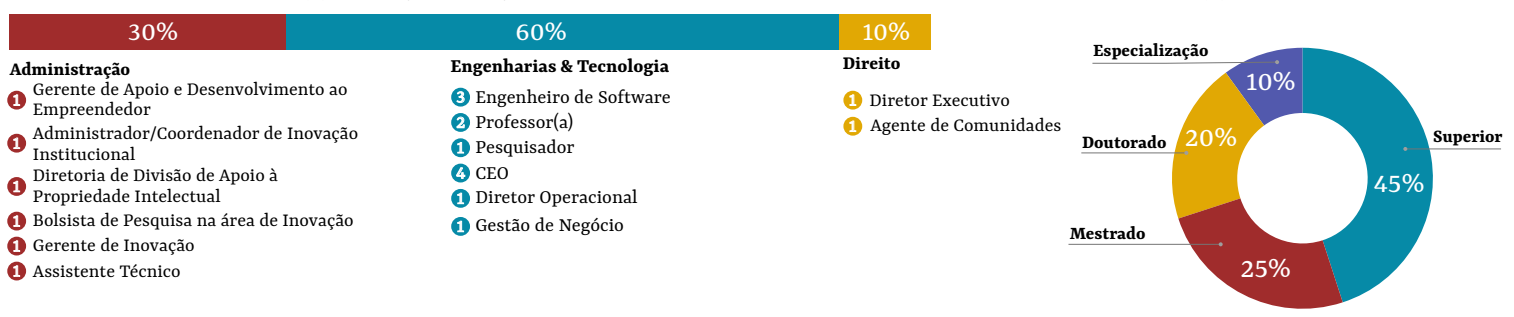

Figura 2. Caracterização dos participantes.

Para que os testes fossem realizados, estabeleceu-se, conforme demonstrado na Figura 3, um roteiro baseado em oito etapas. Em relação à Etapa 6, em específico, definiram-se seis tarefas com as principais funcionalidades da aplicação a serem utilizadas. Destaca-se que, para prover maior realidade à realização das tarefas, o mapa também já contava com algumas entidades e eventos previamente mapeados.

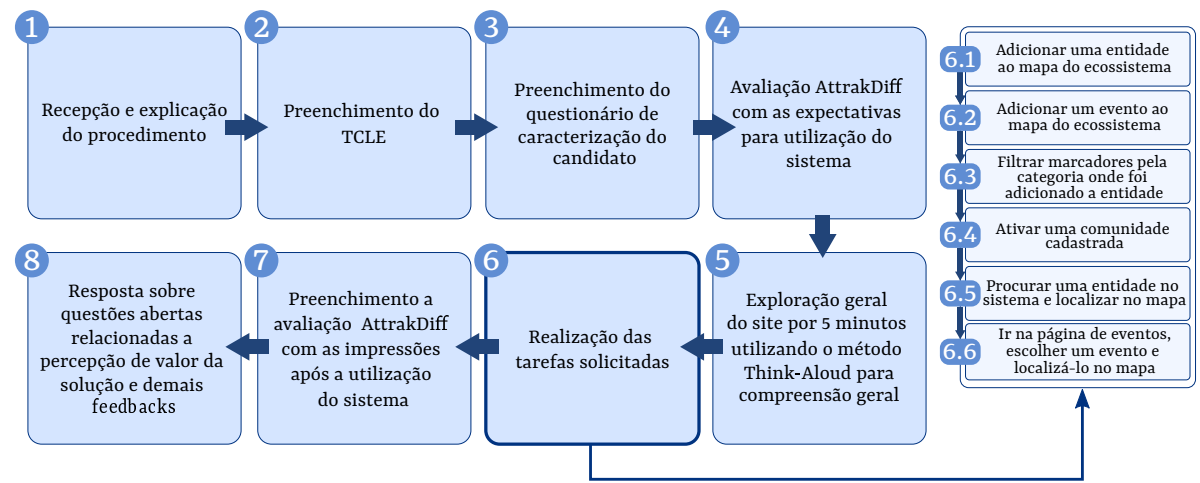

Figura 3. Roteiro dos testes.

Em decorrência do distanciamento social imposto pelo COVID-19, o experimento teve que ser supervisionado de forma online, através da ferramenta de videochamada Zoom ${ }^{4}$. Mesmo remotamente, verificou-se a inocorrência de prejuízos demasiados, haja vista que foi possível observar tanto as reações físicas do usuário pela webcam, quanto a interação com a solução via espelhamento da tela. Ademais, destaca-se que cada teste foi feito de forma individual e que a intervenção do avaliador somente se deu em momentos de solicitação, com o fito de tornar a experiência autêntica. Os testes foram autorizados a serem gravados e transcritos, conforme necessário. Adotou-se, também, a realização de um experimento piloto com um voluntário (designer gráfico com três anos de experiência profissional) para fins de validação do roteiro, porém seu resultado foi descartado. A única melhoria identificada como necessária foi o aprimoramento de scripts de falas para apoio ao pesquisador durante as etapas definidas no roteiro dos testes. Os experimentos duraram entre 30 e 45 minutos, com média de 35 minutos.

\section{Proposta de Mapa Interativo e Colaborativo}

\subsection{Definição do Escopo}

Além da escassez de estudos empíricos sobre elementos colaborativos, de UX e Usabilidade no domínio de mapas de ecossistemas de inovação, notou-se que no estado do

\footnotetext{
4 https://zoom.us/. Acesso em: 04/01/2021.
} 
Ceará não havia nenhum projeto com tal escopo, isto é, intrinsecamente colaborativo e open source. Diante de tal oportunidade, realizou-se previamente uma análise de benchmarking na busca de referências com o propósito de elucidar o escopo para um Produto Mínimo Viável, seja com a definição de requisitos (funcionais e de qualidade) inspirados nos referidos projetos ou a partir de carências identificadas. Conforme explicado anteriormente, três projetos foram selecionados: Minas Gerais, Alagoas e Santa Catarina.

Em termos gerais, definiu-se que o mapa aqui proposto deve funcionar de forma colaborativa, tanto sob a perspectiva de seu desenvolvimento, quanto de seu uso. Neste caso, faz-se necessário que haja a participação de diversos stakeholders, os quais foram classificados em dois tipos: i) a comunidade técnica mantenedora da solução e ii) os usuários finais engajados no ecossistema. Neste momento, sem níveis de permissão, os usuários finais poderão adicionar informações na aplicação, como as entidades do ecossistema, a delimitação das comunidades e a divulgação de eventos. Com isso, as instituições participantes terão suas informações divulgadas de forma acessível e contextualizada. Adicionalmente, também deve ser possível consultar ou filtrar os participantes por meio de categorias. A descrição detalhada dos requisitos, incluindo casos de uso, encontram-se disponíveis para consulta na página de suporte [Castro et al. 2020].

\subsection{Arquitetura do Projeto}

Conforme ilustrado na Figura 4, o modelo arquitetural desenhado neste trabalho adotou o padrão Model-View-Controller, sendo a infraestrutura construída com base na plataforma Firebase. Em particular, utilizou-se Firebase Authentication para gerenciar as autenticações dos usuário da ferramenta, Real Time Database, que é um banco de dados sincronizado em tempo real, especialmente necessário para esta solução, e o Cloud Storage para o armazenamento de imagens. Ademais, adotou-se as linguagens HTML, CSS e JavaScript e, para auxiliar o front-end, optou-se pelo framework Bootstrap. Utilizou-se também a biblioteca jQuery para apoio na manipulação da página. O serviço de mapas foi utilizado via Application Programming Interface do Leaflet, que é uma biblioteca open source JavaScript que facilita 1) gerenciar o mapa da aplicação, 2) adicionar as camadas de conteúdo e 3) gerenciar as interações. Com isso, o Leaflet permite criar camadas de recursos, as quais contém dados vetoriais, como marcadores, linhas, polígonos, entre outros, criar pop-ups, carregar dados de recursos de arquivos GeoJSON (formato para representar recursos geográficos), bem como, personalizá-los e, assim, fazer delimitações de áreas, além de gerir as interações com os componentes do mapa.

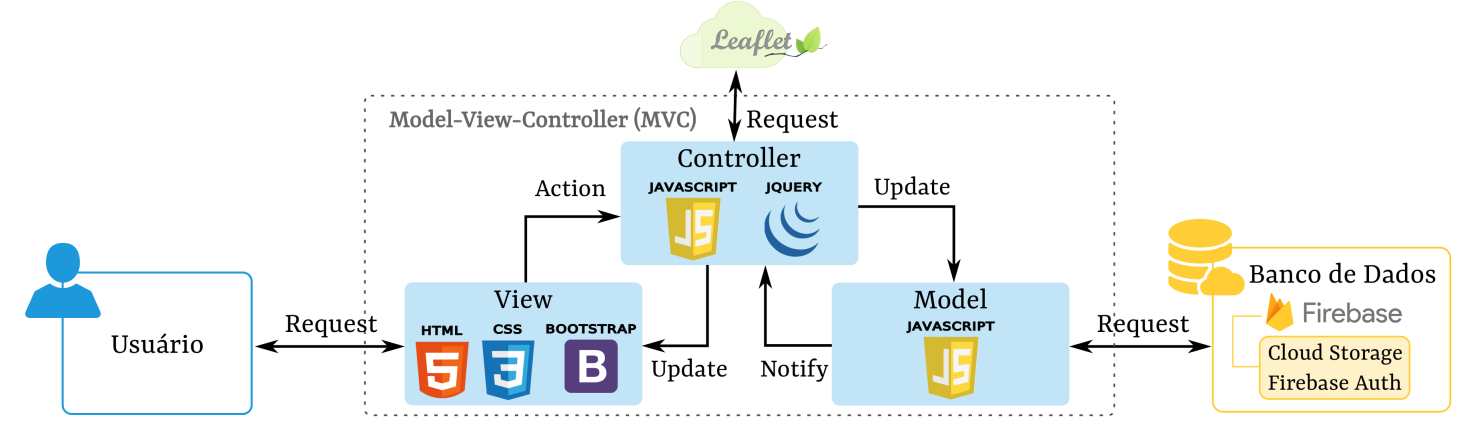

Figura 4. Modelo de arquitetura do projeto. 


\subsection{Demonstração da Solução}

A Figura 5 a apresenta a visão da tela principal com os marcadores exibidos no mapa. No topo, encontra-se a barra de navegação, onde estão contidos os links para as outras páginas do sistema, além dos botões que permitem a filtragem dos marcadores, a adição de novas comunidades e a adição de novos marcadores. Na barra lateral é possível visualizar as categorias de participantes. A Figura 5,b ilustra a página relacionada aos eventos cadastrados pela comunidade. Para visualização das demais telas, sugere-se acessar o link $5^{5}$ da solução, cujo código-fonte encontra-se disponível em um repositório no Github ${ }^{6}$.

(a) Tela Principal

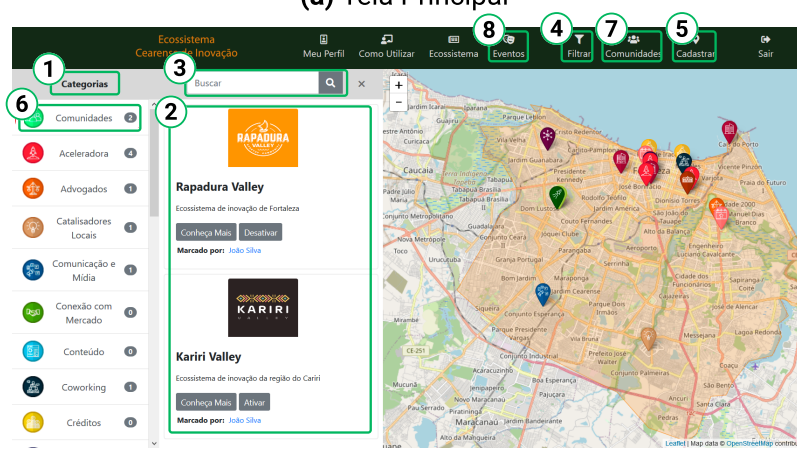

(b) Tela de Eventos

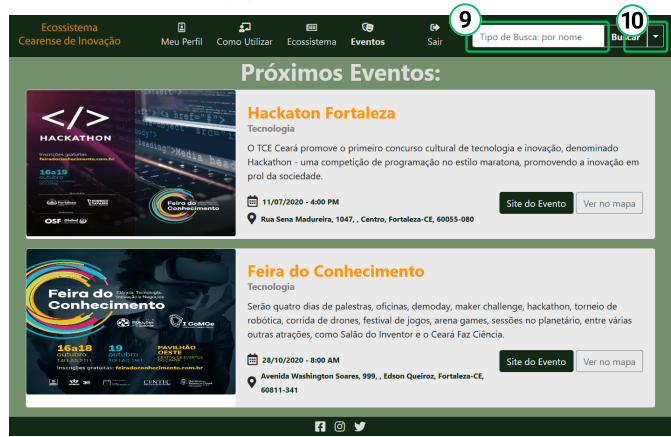

Figura 5. Tela principal e tela de eventos do sistema.

Ao analisar a Figura 5 a, percebe-se que é possível buscar informações dos participantes filtrando-os por categoria no menu lateral esquerdo (1). Tal menu indica as categorias existentes no ecossistema e a quantidade de participantes. Ao clicar em uma categoria, todos os seus respectivos componentes do ecossistema são apresentados (2), sendo possível também acessar o site e acessar sua localização. O sistema possibilita a busca pelo nome do componente do ecossistema no campo de pesquisa (3), caso o usuário deseje. Além disso, pode-se visualizar no mapa apenas as categorias desejadas pelo usuário, através do botão "Filtrar". Por sua vez, para cadastrar um novo participante do ecossistema (5), faz-se necessário clicar em "Cadastrar" na barra de navegação superior e, em seguida, apontar o local do endereço do componente em questão no mapa e clicar no botão "Aqui!" para confirmar o local. Após essa confirmação, irá aparecer uma tela com o formulário de cadastro de componentes. Nesse formulário devem ser preenchidos os dados como nome, site, tipo de local, logotipo e endereço. Em seguida, clicando no botão "Enviar", o procedimento de cadastro de um participante é finalizado, ficando disponível para consulta na tela principal do mapa e no menu lateral da página.

$\mathrm{Na}$ aba Comunidades do menu lateral (6) é possível visualizar as comunidades do ecossistema e destacar a área geográfica ao pressionar o botão "Ativar", ou apagar a área destacada pressionando o botão "Desativar". Para realização do cadastro de comunidades (7) é necessário pressionar o botão "Comunidades" para que um formulário seja aberto e, em seguida, os dados da comunidade, inclusive o código para que seja delimitada a área geográfica desejada, sejam adicionados. No Ceará, por exemplo, as duas maiores comunidades são o Rapadura Valley, que corresponde a comunidade de Forta-

5 https://gesid.github.io/mapa-inovacao. Acesso em: 04/01/2021.

6 https://github.com/gesid/mapa-inovacao. Acesso em: 04/01/2021. 
leza e região, e Kariri Valley, representante da região do Cariri, no sul do estado. O mapeamento de comunidades representa um diferencial idealizado neste projeto.

Para realização do cadastro de eventos, outro diferencial projetado, tem-se um processo similar ao cadastro de participantes, acessada em (5). Ao abrir o formulário de cadastro, pode-se escolher a aba "Evento" para cadastro de eventos. No cadastro de eventos serão solicitados os dados do evento como nome, site, tipo de evento, logo, descrição, data e endereço. Após isso, é necessário clicar no botão "Enviar" para que o evento fique exibível no mapa, bem como na página de eventos do sistema. A página de eventos (Figura 5.b) pode ser acessada pela barra de navegação superior (8). Nela é possível verificar os próximos eventos em formato de lista, incluindo informações associadas, como site do evento ou a localização no mapa. Por fim, na barra superior existe um campo de pesquisa (9), juntamente com um menu (10), onde pode-se escolher o tipo de busca (nome do evento ou pela categoria do evento), facilitando, assim, encontrar eventos de interesse.

\section{Resultados e Análises}

\subsection{Análise à luz do Modelo 4C}

Para análise do projeto à luz do Modelo 4C [Costa et al. 2014], realizou-se uma comparação da solução proposta neste trabalho com as iniciativas selecionadas para benchmarking previamente mencionadas, isto é Minas Gerais, Alagoas e Santa Catarina. Inspirado em [Melo et al. 2017], foi realizada, via observação não participante, uma verificação da quantidade de componentes que dão suporte a cada dimensão que compõem os pilares Comunicação, Coordenação, Colaboração e Cooperação oriundos do Modelo 4C. A Tabela 1 apresenta um resumo comparativo frente às características presentes nas aplicações. Conforme pode-se perceber, a solução aqui proposta contempla a maior quantidade de componentes, incluindo ideias exclusivas diante das demais avaliadas, como a delimitação de comunidades e o cadastro de eventos atrelados à localização. Em segundo lugar, em termos de quantidade de componentes cobertos, vem a solução de Minas Gerais. A iniciativa de Alagoas contempla a menor quantidade de funcionalidades. Em síntese, a presente análise evidencia a aderência da proposta aqui descrita quanto ao Modelo $4 \mathrm{C}$, incluindo os diferenciais incorporados frente a outras soluções identificadas.

\begin{tabular}{|c|c|c|c|c|}
\hline & CE & MG & AL & SC \\
\hline \multicolumn{5}{|l|}{ Ferramentas para comunicação } \\
\hline Perfil - Informações básicas do usuário & $\mathbf{X}$ & $\mathbf{X}$ & & \\
\hline Lista de eventos - Página de exposição de eventos cadastrados e suas informações & $\mathbf{X}$ & $\mathbf{X}$ & & \\
\hline Compartilhamento em mídias sociais & $\mathbf{X}$ & & & $\mathbf{X}$ \\
\hline Mensageiro eletrônico & $\mathbf{X}$ & $\mathbf{X}$ & & \\
\hline Página de como utilizar & $\mathbf{X}$ & & & \\
\hline \multicolumn{5}{|l|}{ Ferramentas para coordenação } \\
\hline Acesso - Identidade - Ferramenta para controle de identidade & $\mathbf{X}$ & & & \\
\hline Bugs/Sugestão - Ferramenta de suporte para reportar bugs e sugestões & $\mathbf{X}$ & $\mathbf{X}$ & & $\mathbf{X}$ \\
\hline Realizar pesquisas simples & $\mathbf{X}$ & $\mathbf{X}$ & $\mathbf{X}$ & \\
\hline Edição de dados do usuário & & $\mathbf{X}$ & & \\
\hline Revisão - Ferramenta de suporte para reportar revisões e denúncias de informações geográficas & $\mathbf{X}$ & $\mathbf{X}$ & & $\mathbf{X}$ \\
\hline \multicolumn{5}{|l|}{ Ferramentas para cooperação e colaboração } \\
\hline GEO - Marcações geográficas & $\mathbf{X}$ & $\mathbf{X}$ & $\mathbf{X}$ & $\mathbf{X}$ \\
\hline GEO - Navegação - Navegação no mapa, visualização das informações e interfaces auxiliares & $\mathbf{X}$ & $\mathbf{X}$ & $\mathbf{X}$ & $\mathbf{X}$ \\
\hline Mural - Acompanhamento customizado na forma de lista & $\mathbf{X}$ & $\mathbf{X}$ & & \\
\hline Filtragem - Filtragem das marcações geográficas & $\mathbf{X}$ & $\mathbf{X}$ & $\mathbf{X}$ & \\
\hline Cadastro de regiões - Cadastro de regiões dentro da região principal & $\mathbf{X}$ & & & \\
\hline Cadastro de eventos - Cadastro de eventos que ocorrerão dentro do ecossiste & $\mathbf{X}$ & & & \\
\hline
\end{tabular}

Tabela 1. Comparativo baseado no Modelo $4 \mathrm{C}$ das iniciativas identificadas. 


\subsection{Análise da Usabilidade}

Baseando-se em [Winckler and Pimenta 2002], solicitou-se aos usuários a realização das seis principais atividades projetadas. Após observar individualmente as gravações de cada participante, contabilizou-se o percentual de acerto destas atividades a partir de três categorias: fácil, caracteriza as atividades que foram realizadas na primeira tentativa; difícil, as atividades que precisaram de mais de uma tentativa; e, sem sucesso, àquelas que o usuário não conseguiu concluir. Conforme informado na Figura 6, verifica-se em média que, em 79\% dos casos, os usuários precisaram somente de uma tentativa para concluir a tarefa. Percebe-se que a funcionalidade com mais facilidade foi a localização de eventos no mapa (6). Por sua vez, a solução que apresentou-se mais difícil foi a de cadastro de evento (2). Conclui-se que, de forma geral, a solução apresentou índices positivos de usabilidade, permitindo uma experiência adequada para as principais atividades delineadas.

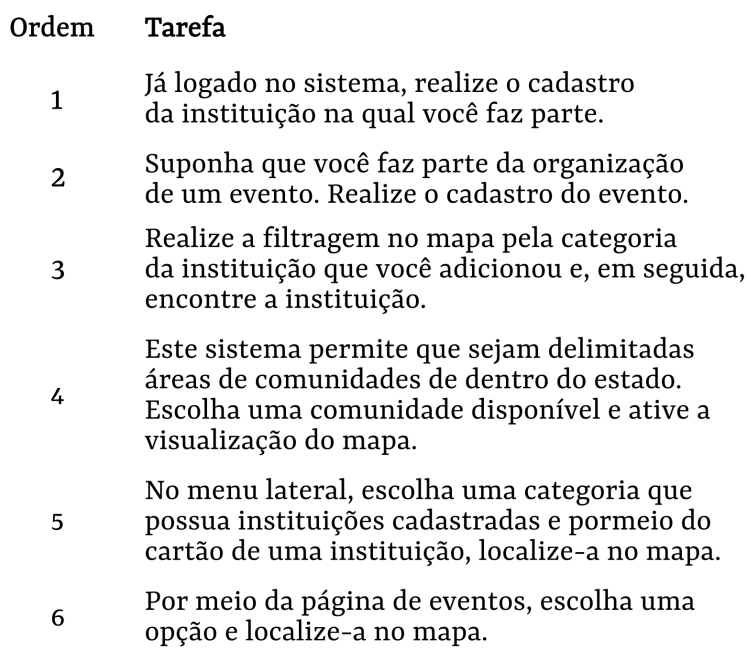
possua instituições cadastradas e pormeio do cartão de uma instituição, localize-a no mapa.

Por meio da página de eventos, escolha uma opção e localize-a no mapa.

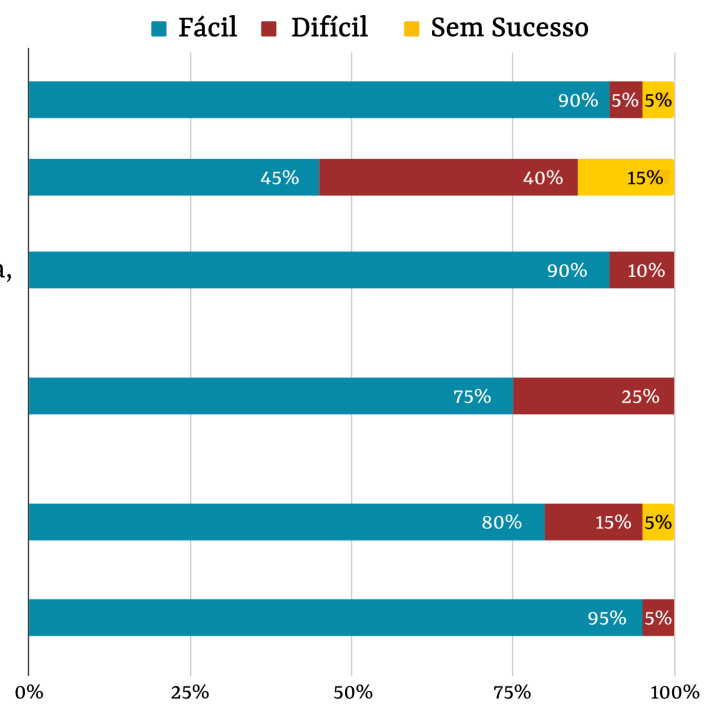

Figura 6. Desempenho na avaliação de usabilidade.

\subsection{Análise da Experiência do Usuário}

O resultado da avaliação de UX, medida por meio do questionário Attrakdiff [Hassenzahl 2004] aplicado pré e pós-utilização da solução, são sintetizados na Figura 7. Na análise das palavras-pares (Figura 7.a), tem-se cada ponto representando os valores médios obtidos para cada tipo de qualidade. A linha azul representa os valores obtidos antes do teste (expectativa), enquanto a linha laranja representa os valores após o teste (realidade). Verifica-se que, apesar da expectativa se demonstrar positiva em todas opções, a experiência também apresentou resultados positivos em quase todos os pontos, com exceção apenas do quesito pouco exigente - desafiador. Nesse caso, em específico, o fato de ser pouco exigente é um resultado almejado e corrobora a análise de usabilidade previamente discutida. Observa-se também um movimento correlacionado nos resultados, com poucas divergências significativas, de modo que em seis pares de palavras houve uma avaliação equiparada. Trazendo uma visão geral dos valores médios para cada dimensão, a Figura 7.b reforça o resultado de que, mesmo inferior à expectativa, a avaliação para todas as dimensões foi positiva, sendo Qualidade Hedônica-Identidade (HQ-I), que reflete o 
nível de identificação do usuário com a aplicação, e Atratividade (ATT) aquelas que apresentaram maior pontuação. Por sua vez, a Qualidade Hedônica-Estímulo (HQ-S), que indica o quão original, estimulante e interessante é a aplicação, apresentou a pontuação mais baixa dentre as quatro dimensões.

(a) Descrição de palavras-pares

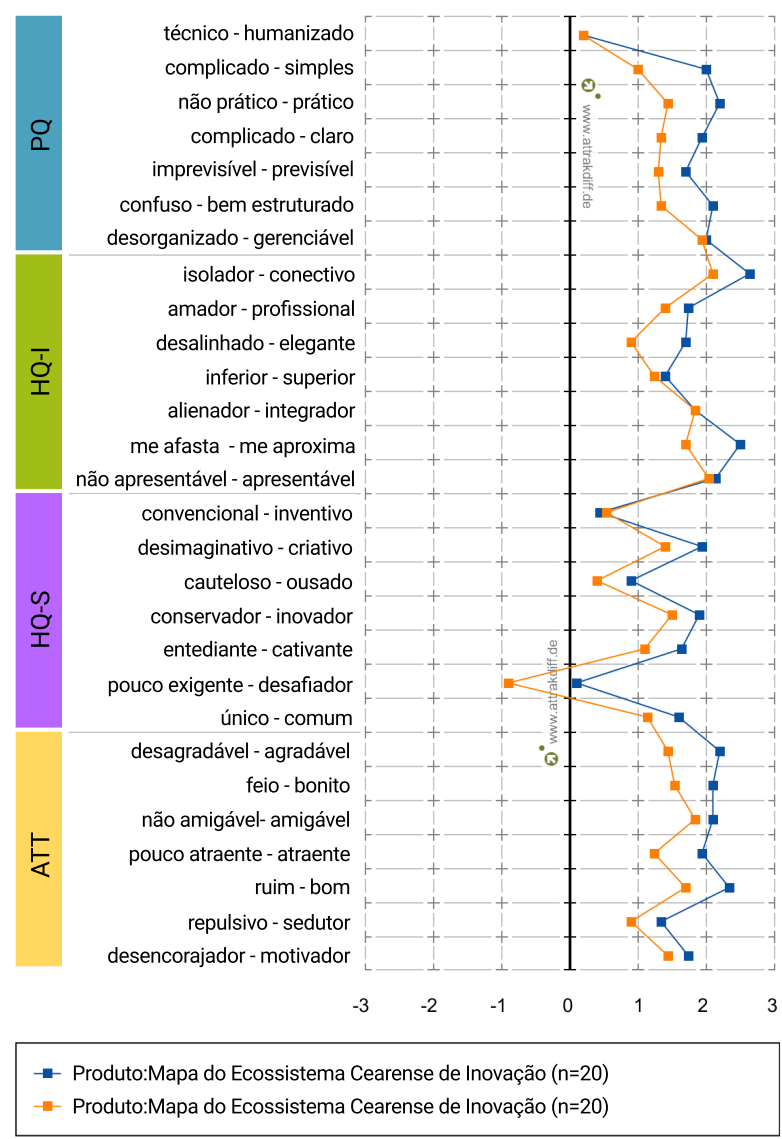

(b) Diagrama de valores médios

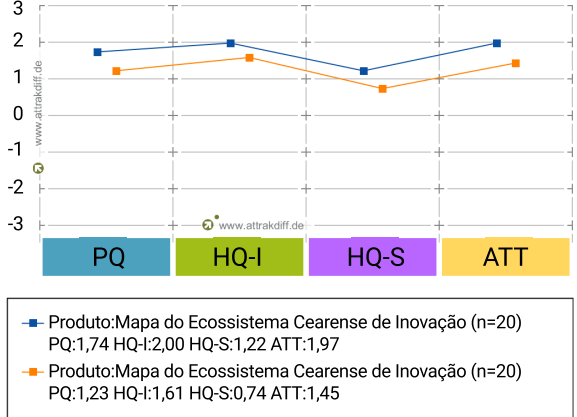

Figura 7. Comparação das avaliações Attrakdiff, pré e pós-utilização.

\subsection{Análise da Percepção de Valor}

Após a execução dos testes, indagou-se três questões de múltipla escolha para avaliação da Percepção de Valor e, em seguida, duas questões abertas cobrindo os pontos positivos e sugestões. Conforme Figura 8, 95\% dos avaliados concordaram que a solução poderia contribuir com a divulgação dos participantes. Em seguida, sobre a utilização, 70\% dos participantes informaram que certamente iriam incluir a sua instituição e $25 \%$ provavelmente incluiriam, totalizando $95 \%$. Na terceira questão, verificou-se que, somando aqueles que "certamente" e "provavelmente" recomendariam a solução, tem-se um total de $95 \%$ dos participantes. Finalmente, obteve-se diversos feedbacks em relação ao layout e a paleta de cores. Segundo um participante "as cores estão um pouco fortes", enquanto outro ressaltou que "o layout está muito conservador, se assemelhando muito aos sites institucionais do governo". Todavia, essa foi uma decisão proposital buscando uma consonância com as plataformas institucionais do estado do Ceará. Como sugestões, destacam-se o aprimoramento da barra de busca para os endereços, melhoria da acessibilidade e facilitar as conexões entre os atores que demandam e que ofertam serviços. 


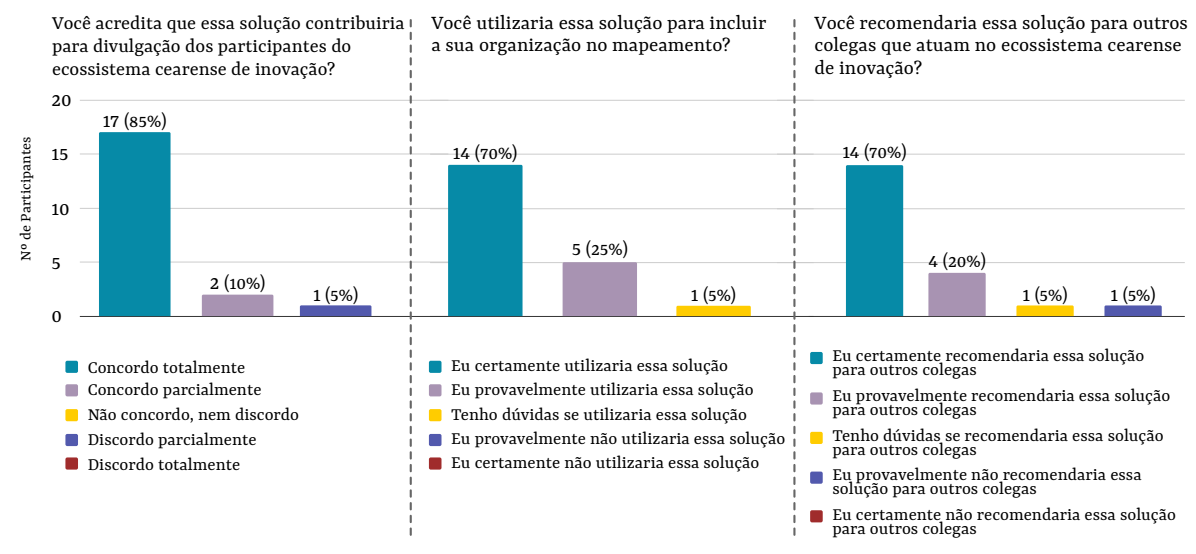

Figura 8. Análise de percepção de valor da solução.

\section{Ameaças à Validade}

Com base em [Wohlin et al. 2012], apresenta-se a seguir ameaças à validade deste estudo. Quanto às ameaças internas, tem-se que cada participante foi submetido a um experimento individual em seu respectivo horário e dia agendado, o que poderia influenciar na interpretação dos testes. Apesar disso, adotou-se um roteiro uniforme de avaliação, validado previamente em teste piloto. Ademais, outros fatores podem ameaçar os resultados, como tratamentos especiais que podem gerar alteração comportamental. O participante saber que está sendo observado também pode influenciá-lo. Para mitigar tais desafios, os participantes receberam uma explicação sobre a abordagem, mas não sobre os pressupostos investigados. Quanto à ameaça externa, destaca-se o número reduzido de participantes. Salienta-se, entretanto, a pluralidade de perfis representando diversos setores. Adicionalmente, segundo [Faulkner 2003], uma amostra com 20 pessoas para um teste de usabilidade, na pior das hipóteses, releva $95 \%$ dos problemas. Considerando as ameaças de conclusão, os 20 participantes não representam todos os perfis existentes no domínio de ecossistemas de inovação. Outra ameaça é a realização do experimento à distância, fora de um ambiente controlado. Por fim, quanto à validade de construção, nota-se que parte dos participantes eram da área da computação, possibilitando um entendimento diferenciado dos testes a partir de seus conhecimentos técnicos prévios. Tal situação, pode induzi-los a um comportamento que não converge com o natural. Em relação à avaliação de Percepção de Valor, utilizou-se a escala de Likert, que apesar de limitar as respostas, complementa em uma perspectiva numérica às duas questões abertas realizadas.

\section{Considerações Finais}

Como contribuições deste trabalho, tem-se a disponibilização de uma solução funcional e open source que permite o mapeamento colaborativo de um ecossistema de inovação, viabilizando assim, a oportunidade que a proposta seja evoluída ou adaptada para outros ecossistemas. Adicionalmente, tem-se o relato de um estudo empírico pioneiro cobrindo elementos colaborativos, de UX e Usabilidade no domínio de ecossistemas de inovação, cuja triangulação dos dados evidenciou resultados positivos quanto ao valor da solução. Tais achados, por sua vez, fomentam perspectivas transversais de trabalhos futuros, vide: como aprimorar, em termos de confiança, o cadastro das entidades; como fomentar o engajamento dos usuários de forma acessível; como potencializar as conexões entre os atores; e analisar a usabilidade da aplicação à luz das Heurísticas de Nielsen. 


\section{Referências}

Bardin, L. (2010). Análise de conteúdo. $4^{\text {th }}$ ed. Lisboa: Edições.

Castro, R., Araújo, A. A., Soares, P., Paixão, M., Maia, P., and Souza, J. (2020). Página de suporte - proposta de mapa interativo e colaborativo para ecossistemas de inovação: Um estudo de caso baseado no estado do ceará. Disponível em: http://gesid.github.io/papers/mapa-inovacao. Acesso em: 04/01/2021.

Costa, A. P., Loureiro, M. J., and Reis, L. P. (2014). Do modelo 3c de colaboração ao modelo 4c: Modelo de análise de processos de desenvolvimento de software educativo. Revista Lusófona de Educação, (27):181-200.

do Nordeste, D. (2020). Ambiente promissor fará ceará avançar na inovação, aponta estudo. Disponível em: https://diariodonordeste.verdesmares.com.br/negocios/ambiente-promissor-fara-ceara-avancar-nainovacao-aponta-estudo-1.2999029. Acesso em: 12/10/2020.

Faulkner, L. (2003). Beyond the five-user assumption: Benefits of increased sample sizes in usability testing. Behavior Research Methods, Instruments, \& Computers, 35(3):379-383.

Hassenzahl, M. (2004). The interplay of beauty, goodness, and usability in interactive products. HumanComputer Interaction, 19(4):319-349.

IPEA (2016). Nota técnica - inovação no brasil: crescimento marginal no período recente. Disponível em: https://www.ipea.gov.br/portal/images/stories/PDFs/nota_tecnica/20161209_nt_34.pdf. Acesso em: 04/01/2021.

Jin-fu, W. (2010). Framework for university-industry cooperation innovation ecosystem: Factors and countermeasure. In 2010 International Conference on Challenges in Environmental Science and Computer Engineering, volume 2, pages 303-306. IEEE.

Lemos, C. (2009). Inovação na era do conhecimento. Parcerias estratégicas, 5(8):157-180.

MacEachren, A. M. and Kraak, M.-J. (2001). Research challenges in geovisualization. Cartography and geographic information science, 28(1):3-12.

Melo, Á., Fernandes, C., Jardim, M., and Barreto, R. (2017). Modelo 3c de colaboração aplicado ao uso de um repositório para o desenvolvimento de interfaces para autistas. In Anais do XIV Simpósio Brasileiro de Sistemas Colaborativos, pages 297-311. SBC.

Rogers, Y., Sharp, H., and Preece, J. (2013). Design de Interação. Bookman Editora.

Schumpeter, J. A. et al. (1939). Business cycles, volume 1. McGraw-Hill New York.

Spinosa, L. and Krama, M. (2014). Ecossistema de inovação e meio urbano: principais desafios para seus gestores.

Tidd, J. and Bessant, J. (2015). Gestão da inovação. $5^{\text {th }}$ ed. Bookman Editora.

Valentim, N. M. C., Silva, W., and Conte, T. (2015). Avaliando a experiência do usuário e a usabilidade de um aplicativo web móvel: Um relato de experiência. In XVIII Congresso Ibero-Americano em Engenharia de Software (CIbSE), page 788.

Wazlawick, R. S. (2009). Metodologia de pesquisa para ciência da computação. Elsevier.

Winckler, M. and Pimenta, M. S. (2002). Avaliação de usabilidade de sites web. In Anais da Escola de Informática da SBC SUL (ERI). Porto Alegre: Sociedade Brasileira de Computação (SBC), volume 1, pages $85-137$.

Wohlin, C., Runeson, P., Höst, M., Ohlsson, M. C., Regnell, B., and Wesslén, A. (2012). Experimentation in software engineering. Kluwer Academic Publishers, Norwell, MA, USA.

Yin, R. K. (2017). Case study research and applications: Design and methods. Sage publications. 